\title{
Cytokine Expression in Dengue Fever and Dengue Hemorrhagic Fever Patients with Bleeding and Severe Hepatitis
}

\author{
Hisham Ahmed Imad, ${ }^{1}$ Weerapong Phumratanaprapin, ${ }^{1 \star}$ Benjaluck Phonrat, ${ }^{1}$ Kesinee Chotivanich, ${ }^{1}$ \\ Prakaykaew Charunwatthana, ${ }^{1}$ Sant Muangnoicharoen, ${ }^{1}$ Srisin Khusmith, ${ }^{2}$ Terapong Tantawichien, ${ }^{3}$ Juthamas Phadungsombat, ${ }^{4}$ \\ Emi Nakayama, ${ }^{4,5}$ Eiji Konishi, ${ }^{6}$ and Tatsuo Shioda ${ }^{4,5}$ \\ ${ }^{1}$ Department of Clinical Tropical Medicine, Faculty of Tropical Medicine, Mahidol University, Bangkok, Thailand; ${ }^{2}$ Department of Microbiology and \\ Immunology, Faculty of Tropical Medicine, Mahidol University, Bangkok, Thailand; ${ }^{3}$ Division of Infectious Diseases, Department of Medicine, \\ Chulalongkorn University, Bangkok, Thailand; ${ }^{4}$ Mahidol-Osaka Center for Infectious Diseases, Faculty of Tropical Medicine, Mahidol University, \\ Bangkok, Thailand; ${ }^{5}$ Research Institute for Microbial Diseases, Osaka University, Osaka, Japan; ${ }^{6}$ BIKEN Endowed Department of Dengue Vaccine \\ Development, Faculty of Tropical Medicine, Mahidol University, Bangkok, Thailand
}

\begin{abstract}
Dengue is the most common mosquito-borne flaviviral infection in the world today. Several factors contribute and act synergistically to cause severe infection. One of these is dysregulated host immunological mediators that cause transient pathophysiology during infection. These mediators act on the endothelium to increase vascular permeability, which leads to plasma leakage compromising hemodynamics and coagulopathy. We conducted a prospective study to explore the expression of pro- and anti-inflammatory cytokines and how they relate to clinical dengue manifestations, by assessing their dynamics through acute dengue infection in adults admitted to the Hospital for Tropical Diseases, Bangkok, Thailand. We performed cytokine analysis at three phases of infection for 96 hospitalized adults together with serotyping of confirmed dengue infection during the outbreaks of 2015 and 2016 . The serum concentrations of seven cytokines (interleukin [IL]-2, IL-4, IL-6, IL-8, IL-10, tumor necrosis factor alpha, and interferon gamma) were measured in duplicate using a commercial kit (Bio-Plex Human Cytokine Assay). In this study, the cytokine profile was suggestive of a T-helper 2 response. Most patients had secondary infection, and the levels of viremia were higher in patients with plasma leakage than those without plasma leakage. In addition, we observed that bleeding and hepatitis were associated with significantly higher levels of IL-8 during the early phases of infection. Furthermore, IL-6 levels in the early phase of infection were also elevated in bleeding patients with plasma leakage. These results suggest that IL-6 and IL-8 may act in synergy to cause bleeding in patients with plasma leakage.
\end{abstract}

\section{INTRODUCTION}

The dengue virus is a flavivirus of the family Flaviviridae and has been characterized into four serotypes (Dengue viruses 1 to 4). Transmitted by Aedes species, dengue infection is the most commonly occurring arthropod-borne viral infection globally. Several factors such as globalization, urbanization, and lack of effective vector control have facilitated the spread of this disease beyond the subtropics. ${ }^{1}$

It is estimated that 50-400 million infections occur annually. In Thailand, the first epidemic of dengue hemorrhagic fever (DHF), a severe form of dengue virus infection, occurred in 1958. Multiple outbreaks with greater epidemic potential have occurred since then, with a range of 15,000-105,000 cases occurring each year in Thailand. ${ }^{2}$ The infection causes a systemic viral infection, and complications are associated with the pathogenic role of endothelial cells. ${ }^{3-5}$ Cytokines $^{6,7}$ increase vascular permeability and hemorrhage during dengue infection. These molecules are proteinaceous and are secreted during innate and adaptive immunological responses, acting as inflammatory mediators or modulatory molecules during dengue infection. Viral infection activates the innate immune response to express pro-inflammatory cytokines that recruit and activate cells involved in inflammation and the induction of adaptive immunity. Cytokines also help distinguish between T-helper 1 and T-helper 2 (Th2) cells, and their expression in dengue has been reviewed extensively. ${ }^{8}$ The dengue virus is tropic and replicates in human monocytes, macrophages, and hepatocytes, which are known to express cytokines. ${ }^{9}$

* Address correspondence to Weerapong Phumratanaprapin, Faculty of Tropical Medicine, 420/6 Rajchawithi Rd., Bangkok 10400, Thailand. E-mail: weerapong.phu@mahidol.ac.th
The endothelium plays a pivotal role in the pathophysiology of dengue infection. ${ }^{6}$ Activation of the endothelium by the expression of interleukin (IL)-6, IL-8, and tumor necrosis factor alpha (TNFa) have been previously described.,10 This activation of the endothelial system not only contributes to the bleeding, which is common in dengue fever (DF) and DHF, ${ }^{11}$ but also to liver damage, leading to hepatitis complicating dengue. $^{12}$ Bleeding manifestations are seen in $20-60 \%{ }^{13}$ of cases, and hepatitis with a 100-fold increase in transaminase was reported in $7 \%$ of cases. ${ }^{14}$ Thrombocytopenia with impairment of the coagulation system is associated with bleeding in dengue, ${ }^{15}$ and multiple factors contribute to the development of hepatitis. These factors include hypoxic injury caused by decreased perfusion, direct damage by the virus, and dysregulated immune-mediated injury in response to dengue virus. Interleukin8 is expressed by hepatocytes during dengue infection, ${ }^{16}$ with reports of cytokines correlating with elevated transaminase levels $^{17}$ and the expression of TNFa following the apoptosis of hepatocytes in dengue infection. ${ }^{18}$ Others reported a correlation between severity and IL-6 and TNFa levels. ${ }^{19}$ However, only limited data are available for the relationship between the increased expressions of cytokines with hepatitis and bleeding. In the present study, we investigated the expressions of cytokines during dengue infection with bleeding and hepatitis and assessed the degree of viremia with plasma leakage.

\section{MATERIALS AND METHODS}

This was a prospective study of hospitalized adults with symptomatic dengue infection at the Hospital for Tropical Diseases, Bangkok, Thailand. Ethical approval to conduct this study was obtained from the Ethics Committee, Faculty of Tropical Medicine, Mahidol University. 
TABLE 1

Demographic data, clinical findings, and laboratory parameters in dengue infection

\begin{tabular}{|c|c|c|c|}
\hline Parameters & Dengue fever $=59, n(\%)$ & Dengue hemorrhagic fever $=37, n(\%)$ & $P$-value \\
\hline Females & $20(33.89)$ & $18(48.64)$ & 0.2 \\
\hline Males & $39(66.10)$ & 19 (51.35) & \\
\hline Age (years), mean \pm SD & $31 \pm 11$ & $33 \pm 11$ & 0.44 \\
\hline Primary infection, age mean \pm SD & $25 \pm 11$ & 0 & NA \\
\hline Secondary infection, age mean \pm SD & $32 \pm 11$ & $33 \pm 11$ & 0.11 \\
\hline Duration of illness, mean $\pm S D$ & $6.95 \pm 0.9$ & $7.8 \pm 0.8$ & 0.01 \\
\hline Fever & $59(100)$ & $37(100)$ & NA \\
\hline Headache & $53(89.83)$ & $37(100)$ & 0.02 \\
\hline Myalgia/arthralgia & 43 (72.88) & 34 (91.89) & 0.03 \\
\hline Nausea/vomiting & $29(49.15)$ & $20(54.05)$ & 0.75 \\
\hline Lymphadenopathy & 21 (35.59) & $22(59.45)$ & 0.02 \\
\hline Hepatomegaly & $18(30.50)$ & $15(40.50)$ & 0.31 \\
\hline \multirow[t]{2}{*}{ Bleeding } & $20(32.78)$ & $20(54.05)$ & 0.05 \\
\hline & Mean \pm SD & Mean \pm SD & $P$-value \\
\hline Hemoglobin (g/dL) & $14.3 \pm 1.3$ & $14.2 \pm 1.9$ & 0.53 \\
\hline Hematocrit $(\%)$ & $42.4 \pm 3.8$ & $41.9 \pm 5.3$ & 0.54 \\
\hline Leukocytes $\left(10^{3} / \mu \mathrm{L}\right)$ & $3.6 \pm 1.7$ & $3.4 \pm 1.5$ & 0.5 \\
\hline Neutrophils (\%) & $50.7 \pm 16.5$ & $51.3 \pm 16.8$ & 0.85 \\
\hline Lymphocytes (\%) & $26.7 \pm 12.5$ & $25.3 \pm 11.6$ & 0.58 \\
\hline Platelets $\left(10^{3} / \mu \mathrm{L}\right)$ & $81 \pm 46$ & $63 \pm 43$ & 0.06 \\
\hline Atypical lymphocytes (\%) & $14 \pm 10$ & $13 \pm 12$ & 0.75 \\
\hline Sodium (mmol/L) & $136 \pm 3$ & $134 \pm 2$ & 0.06 \\
\hline Potassium (mmol/L) & $4.2 \pm 3.9$ & $3.7 \pm 0.4$ & 0.85 \\
\hline Bicarbonate $(\mathrm{mmol} / \mathrm{L})$ & $23.7 \pm 2.3$ & $23.9 \pm 2.9$ & 0.71 \\
\hline Blood urea nitrogen (mg/dL) & $10.1 \pm 5.0$ & $11.2 \pm 4.6$ & 0.23 \\
\hline Creatinine (mg/dL) & $0.9 \pm 0.3$ & $0.9 \pm 0.3$ & 0.9 \\
\hline Total protein (g/dL) & $6.9 \pm 0.5$ & $8.9 \pm 12.7$ & 0.25 \\
\hline Albumin $(\mathrm{g} / \mathrm{dL})$ & $3.9 \pm 0.3$ & $3.9 \pm 0.5$ & 0.53 \\
\hline Total bilirubin (mg/dL) & $0.5 \pm 0.2$ & $0.6 \pm 0.5$ & 0.14 \\
\hline Direct bilirubin (mg/dL) & $0.19 \pm 0.1$ & $0.3 \pm 0.3$ & 0.16 \\
\hline Aspartate transaminase (U/L) & $224 \pm 399$ & $308 \pm 528$ & 0.24 \\
\hline Alanine transaminase (U/L) & $127 \pm 192$ & $122 \pm 184$ & 0.93 \\
\hline
\end{tabular}

During the dengue outbreak in 2015 and 2016, we recruited adults who presented within 5 days after developing symptoms and with a positive non structural protein 1 antigen or a positive anti-dengue lgM test into our study. We excluded any vulnerable groups and participants who had received a transfusion of blood products during the study period. Because this was an observational study, all consenting participants meeting the study criteria were recruited during the 2year study period. The participants were grouped based on the WHO 1997 classification, and a chest X-ray was performed to detect pleural effusion. Complications in dengue

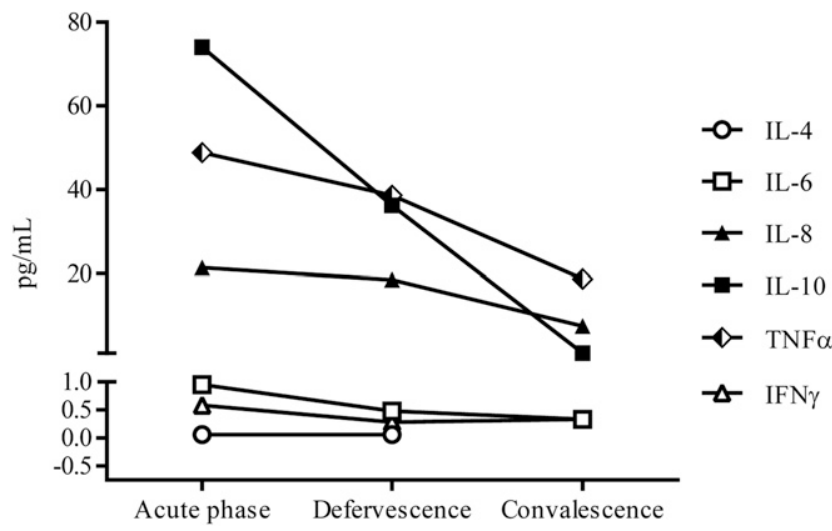

FIGURE 1. Cytokine expression during the three phases of dengue infection. infection included bleeding and severe hepatitis (aspartate aminotransferase/alanine aminotransferase $>400 \mathrm{U} / \mathrm{L}$ ).

Cytokine measurement. Blood specimens were collected for cytokine analysis during the three phases of infection. A commercial assay (Bio-Plex Human Cytokine Assay; Bio-Rad Inc., Hercules CA) was performed to detect the levels of IL-2, IL-4, IL-6, IL-8, IL-10, interferon gamma (IFNy), and TNFa. In brief, sera obtained from participants during the three phases were mixed with beads coated with antibodies to cytokines and a unique fluorescent intensity. Subsequently, the mixtures were incubated with biotinylated anti-cytokine antibodies. Finally, phycoerythrin-conjugated streptavidin was added, and the fluorescent signals were detected using a multiplex array reader (Bio-Plex 200 System, Bio-Rad Inc., Hercules, CA). Raw data were initially measured as the relative fluorescence intensity and then converted to cytokine concentration based on a standard curve generated from the reference concentrations.

The acute phase specimen corresponded to blood collected within 5 days following the development of symptoms of dengue infection. The defervescence specimens were obtained 8 hours after the participants had remained afebrile with a recorded body temperature of $37.7^{\circ} \mathrm{C}$ or lower. The convalescence specimens were obtained during the follow-up within or later than 2 weeks after the onset of symptoms. The standard provided by the manufacturer was used as a control, and the observed readings were determined as the detection limits in $\mathrm{pg} / \mathrm{mL}$. 


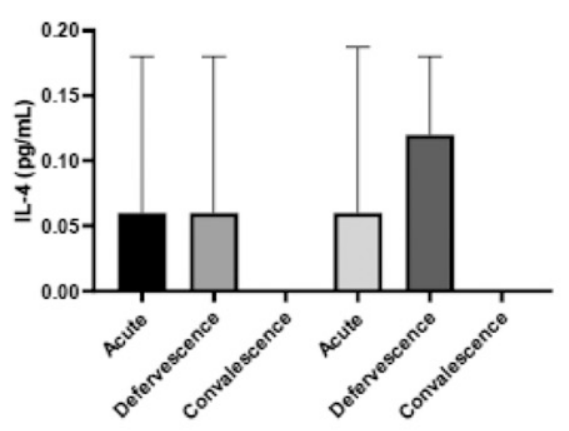

DF

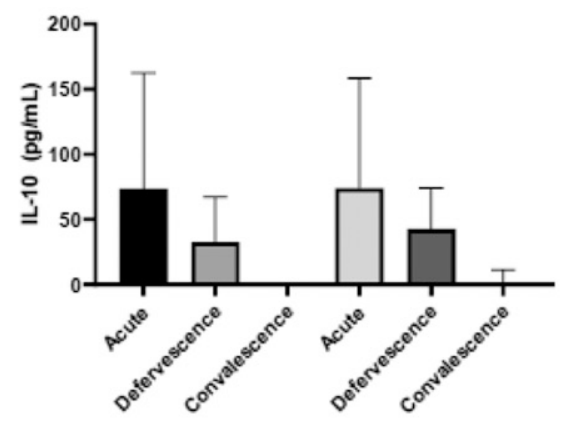

DF

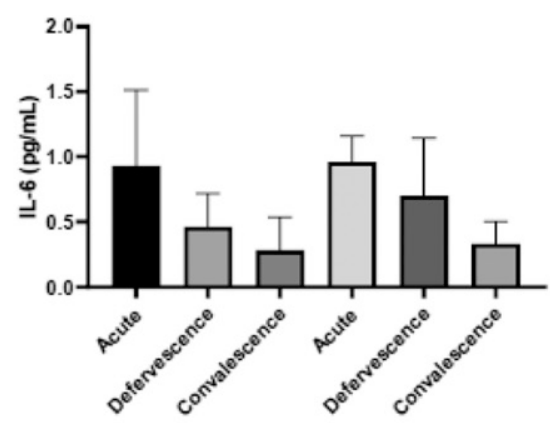

DF

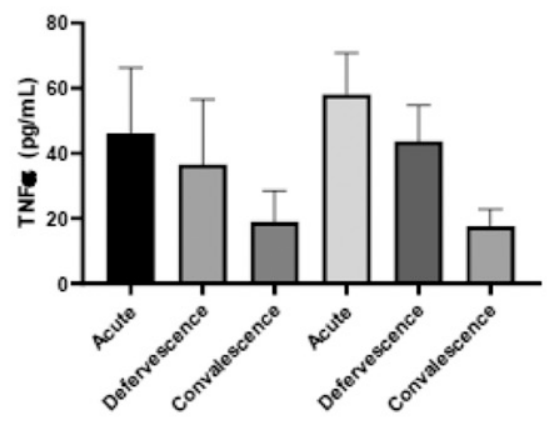

DF

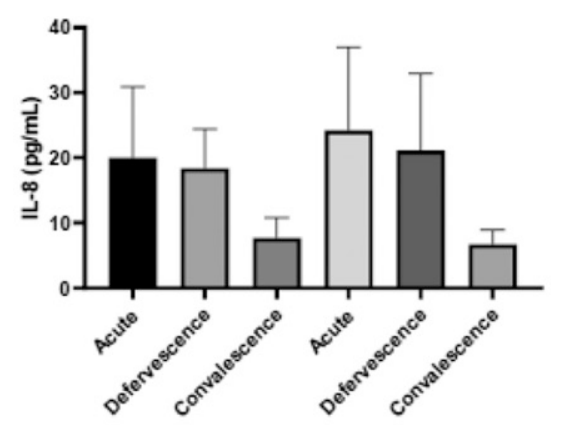

DF

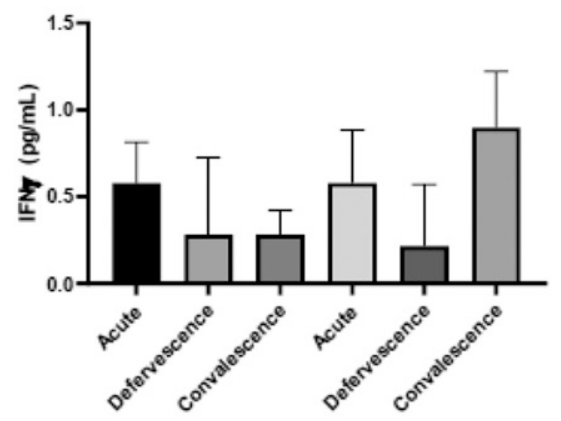

DF

DHF

FIGURE 2. Cytokine profile during the three phases of infection dengue fever vs. dengue hemorrhagic fever.

Quantification of RNA and sequence of infection. To determine the dengue serotype, a commercially available dengue subtyping multiplex kit by the Genesig company (Chandler's Ford, United Kingdom) was used as per the manufacturer's instruction. ${ }^{20}$ Then, RNA quantification was performed using a One-Step SYBR ${ }^{\circledR}$ Prime Script RT-PCR Kit II, Takara Bio Inc., (Kusatsu, Japan); cDNA synthesis from RNA was performed using reverse transcriptase Prime Script RTase; and polymerase chain reaction amplification was performed by TaKaRa Ex Taq HS. This technique was previously described. ${ }^{21}$ For dengue serology, commercially available dengue $\operatorname{lgM}$ and $\operatorname{lgG}$ immunochromatography kits from Panbio Dengue Duo Cassette Abbott Inc., (Chicago, IL) were used in accordance with the manufacturer's protocol. A serum aliquot representing the day of defervescence was used in these test kits. Primary infections were diagnosed

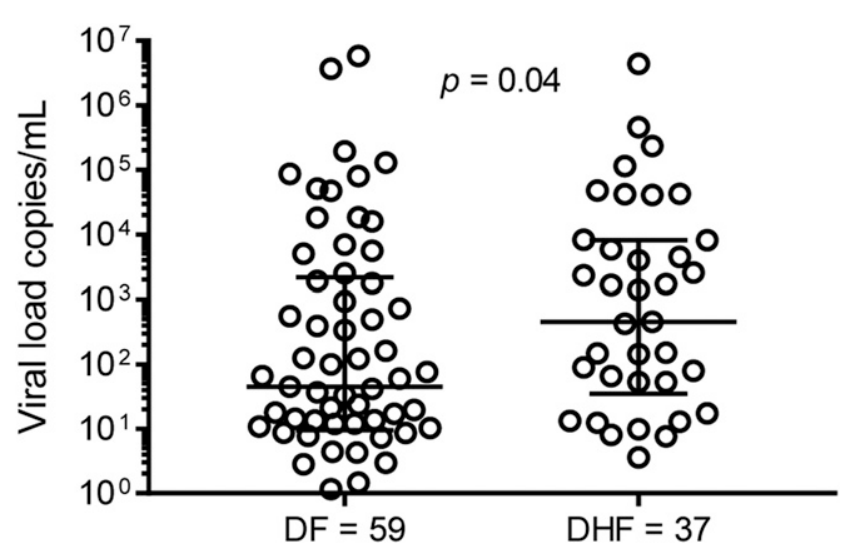

FIGURE 3. Viral load during the acute phase in dengue fever and dengue hemorrhagic fever. when only anti-dengue lgM was positive together with the appearance of the control band. Secondary infections were determined when both anti-dengue $\lg \mathrm{M}$ and $\lg \mathrm{G}$ were positive, including the appearance of the control band.

Statistical analysis. The Mann-Whitney $U$-test was used to assess differences in the cytokine levels between DF and DHF. To establish the correlation between cytokine levels and clinical parameters/findings, a correlation matrix was applied. Results are given as the correlation coefficient; $r$ (range, from -1 to +1 ). A two-tailed $P$-value $<0.05$ was considered significant for all tests performed. One-way analysis of variance was used to evaluate differences among cytokine levels in the different groups. All statistical analyses were performed using Microsoft Excel, SPSS version 18, and GraphPad Prism 7 for Windows, version 7.30 (San Diego, CA).

\section{RESULTS}

In this study, 96 hospitalized adult patients with a confirmed dengue infection were recruited during the outbreak in 2015 and 2016. The demographic and clinical data as well as laboratory parameters of the study population and clinical details are shown in Table 1. Our results indicate that symptoms of systemic illness including headache $(P=0.02)$, myalgia $(P=0.03)$, lymphadenopathy $(P=0.02)$, and bleeding $(P=0.05)$ were more common in DHF. A longer duration of hospitalization was observed in DHF cases than DF cases $(P=0.01)$. The hematological profile was typical for dengue infection, with leukopenia and thrombocytopenia (platelet count $<100 \times 10^{3} / \mu \mathrm{L}$ ) being obvious findings in our subjects. From the analysis of liver function tests, transaminitis was observed in $11 \%$ of subjects. From the analysis of liver function tests, transaminitis was observed in $11 \%$ of 


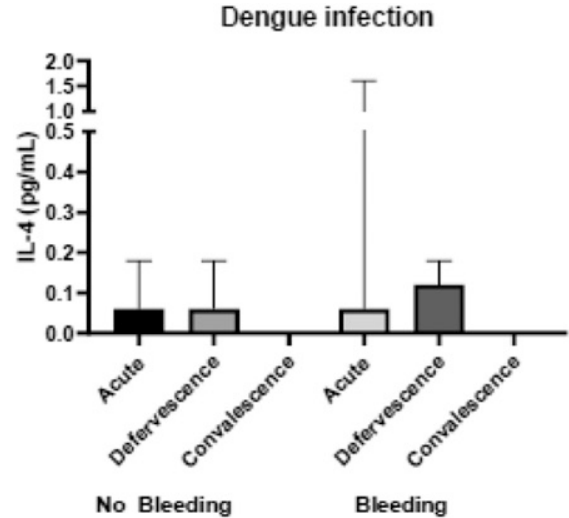

Dengue Infection

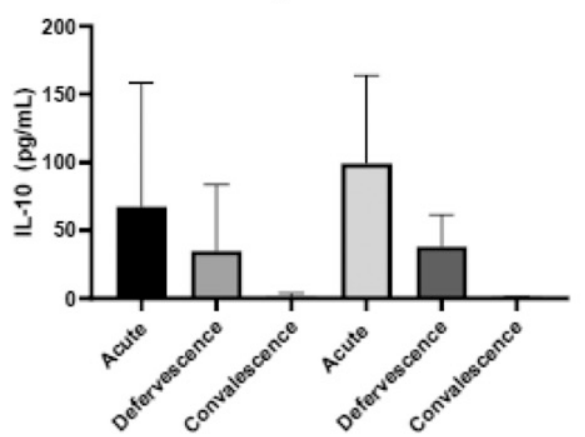

No Bleeding

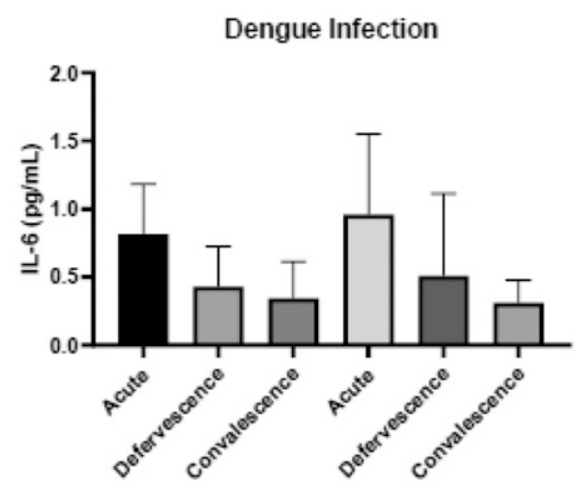

No Bleeding

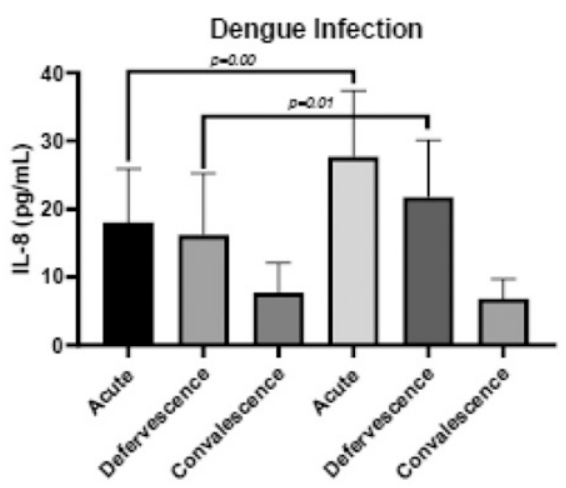

No Bleeding
Bleeding
Dengue Infection

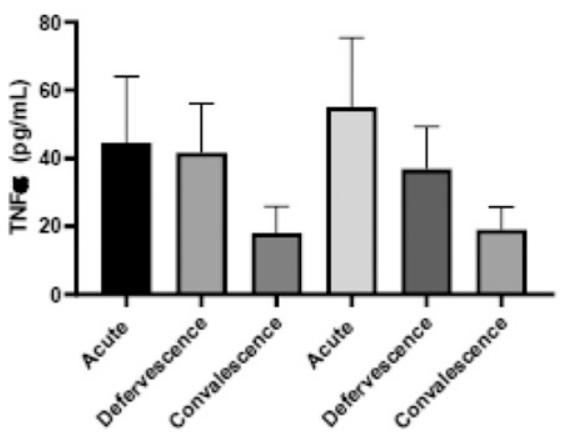

No Bleeding

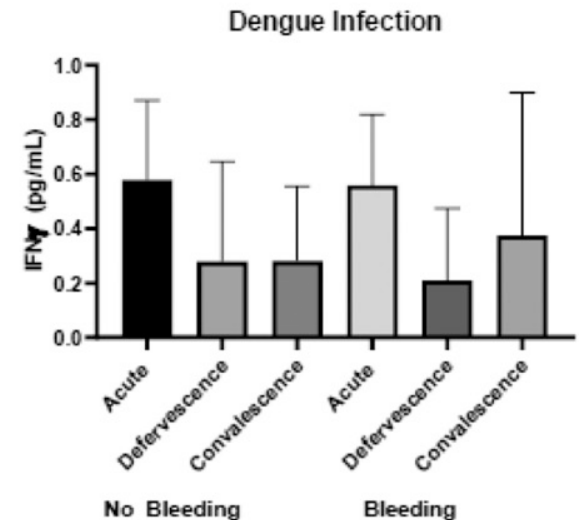

FIGURE 4. Cytokine profile for bleeding during the three phases of infection.

subjects. The mean \pm SD levels of aspartate aminotransferase and alanine aminotransferase for DF were $224 \pm 399 \mathrm{U} / \mathrm{L}$ and $127 \pm 192 \mathrm{U} / \mathrm{L}$, and for DHF were $308 \pm 528 \mathrm{U} / \mathrm{L}$ and $122 \pm$ $184 \mathrm{U} / \mathrm{L}$, respectively.

Cytokine expressions during dengue infection. Figure 1 shows the trend of the expressions of IL-4, IL-6, IL-8, IL-10, TNFa, and IFNy during the three phases of dengue infection. The expressed cytokines peaked during the acute phase. Interleukin-10 was most expressed during the acute phase followed by TNFa, IL-8, IL-6, IFNy, and IL-4. Interleukin-2 was not detectable. At defervescence, TNFa was the most expressed, and during convalescence, IL-4 had diminished to low levels and was undetectable in most cases. We investigated the cytokine profile by grouping our subjects based on their clinical classification. These included 59 subjects with DF and 37 with DHF, of whom 17 were of DHF grade I, 19 were of DHF grade II, and a single participant was of DHF grade IV as per the WHO 1997 classification. ${ }^{22}$ Most patients had secondary dengue infection and had an uneventful milder clinical course during hospitalization. Complications identified in our study were shock in a single subject, and bleeding in $42 \%$ and hepatitis in $11 \%$ of study subjects. The bleeding manifestations were mucosal bleeding in $31.6 \%$, menorrhagia in $27.5 \%$, epistaxis in $5.1 \%$, melena in two subjects, and hematemesis in a single subject. Our results revealed no significant differences in cytokine levels during the three phases of infection between subjects with and without plasma leakage (Figure 2). Nevertheless, we did observe higher viremia in those with plasma leakage (Figure 3).
Cytokines profile with bleeding. Interleukin-8 expression was significantly elevated during the acute phase and at defervescence. The IL-8 median value (interquartile range) was 27.65 (range, 17.42-37.42) pg/mL for the group with bleeding during the acute phase and 17.98 (range, 13.06-25.95) pg/mL for the group without bleeding $(P=0.00$, Figure 4). Similarly, at defervescence, the IL-8 median value (IQR) was 21.76 (range, 16.86-30.14) $\mathrm{pg} / \mathrm{mL}$ for the group with bleeding and 16.19 (range, 11.18-25.30) pg/mL for the group without bleeding $(P=0.01$, Figure 4). These data represent the detectable levels of cytokines in 96 subjects during the three phases of dengue infection. The cytokine levels are shown as the median and IQR in $\mathrm{pg} / \mathrm{mL}$. The Mann-Whitney U-test was used to determine the $P$ value between two groups using the median value.

Similarly, the levels of IL-8 were significantly higher in DHF grade II-IV patients than DHF grade I patients (Figure 5). In addition, IL-6 levels during the acute phase were significantly elevated in DHF grade II-IV compared with DHF grade I (Figure 5). The levels of IL-8 were also significantly higher in DF with bleeding than DF without bleeding during the acute phase of infection (Figure 6).

Cytokine profile with hepatitis. In the cytokine profile generated for severe hepatitis (AST/AST > $400 \mathrm{U} / \mathrm{L}$ ), we observed that in addition to IL-8 being significantly elevated during the acute phase $(P=0.00)$ and at defervescence $(P=$ $0.01)$, TNFa was significantly elevated during the acute phase $(P=0.01$, Figure 7).

The levels of IL-8 were significantly higher during the acute phase and at defervescence in patients with an increase in 
DHF

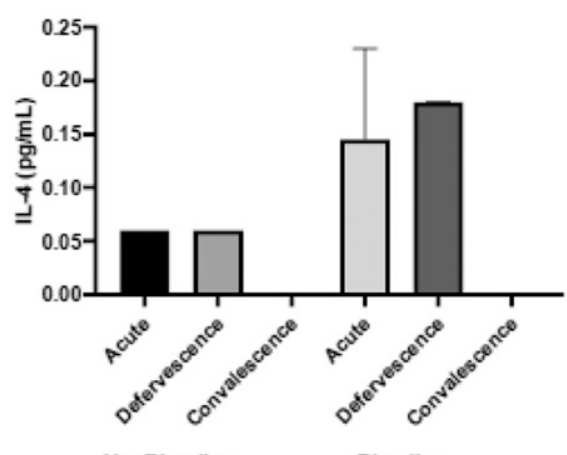

No Bleeding

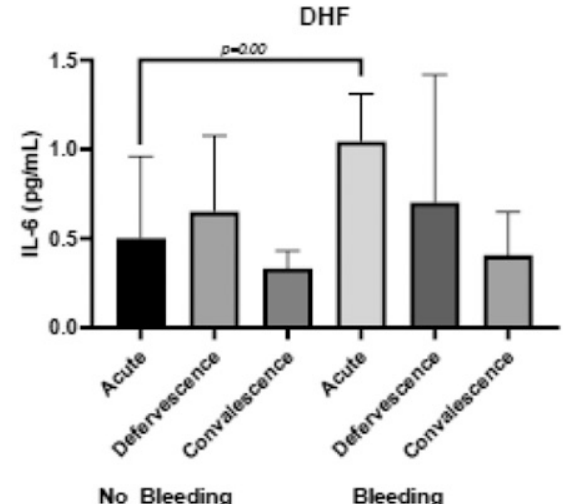

DHF

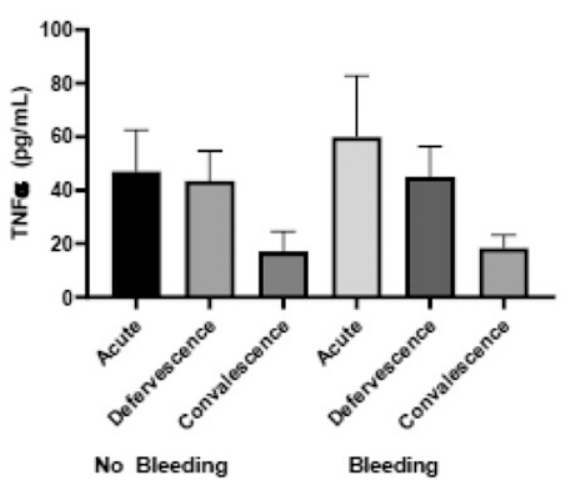

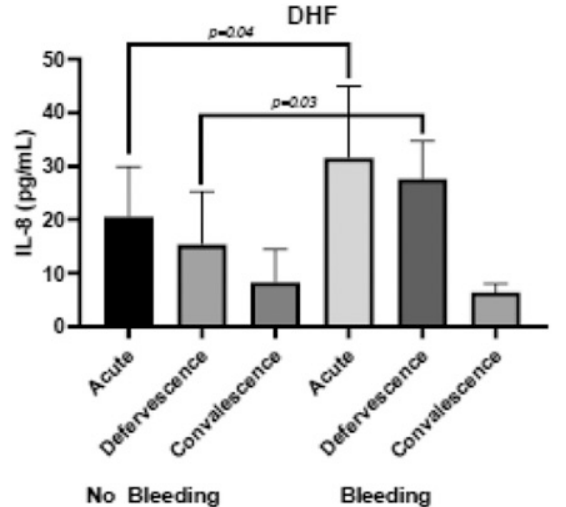

DHF

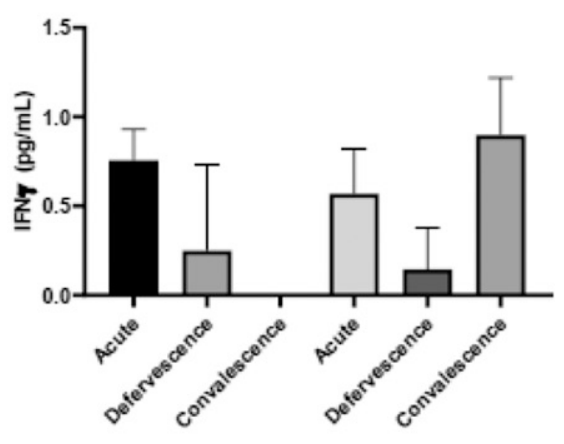

No Bleeding

Bleeding

FIGURE 5. Cytokine profile for bleeding in dengue hemorrhagic fever during the three phases of infection.

liver enzymes (AST/ALT $\geq 1,000 \mathrm{U} / \mathrm{L})$. The median value of IL-8 was $30.28 \mathrm{pg} / \mathrm{mL}$ (IQR, 25.18-86.58) in subjects with AST/ALT $\geq 1,000 \mathrm{U} / \mathrm{L}$, which was significantly elevated during the acute phase $(P=0.03)$. At defervescence, in subjects with $A S T / A L T \geq 1,000 \mathrm{U} / \mathrm{L}$, the median IL-8 value was $32.29 \mathrm{pg} / \mathrm{mL}$ (IQR, 23.80-57.67), which was significantly elevated compared with subjects with AST/ALT $<1,000 \mathrm{U} / \mathrm{L}$ $(P=0.01)$.

\section{DISCUSSION}

In the present study, the levels of cytokines during dengue infection were measured, and we analyzed the profiled cytokine patterns to determine the immunopathophysiological events that occur during dengue infection leading to complications, such as bleeding and hepatitis. To the best of our knowledge, few studies have performed cytokine analysis during the three phases of infection. In particular, we designed our study to measure the cytokine levels at defervescence and identified elevated cytokine levels with complications. In viral infections, cytokines can interfere with the interferon signaling pathway, ${ }^{23}$ which is the host's primary antiviral response to an acute infection. ${ }^{24}$ Our study subjects had a diminished IFNy response, which might have resulted from a secondary dengue infection, where the Th1 response is prominent in primary infection. ${ }^{25}$ This phenomenon was previously demonstrated in vitro, where dengue virus-infected cells failed to express or demonstrated a reduced expression of IFNy on secondary infection. ${ }^{26}$ The cytokine response reflecting a T-helper 2 response was supported by our undetectable levels of secreted IL-2, in addition to the very low levels of IFNy, with an increased expression of IL-10. This trend of cytokine expression also suggests immunoregulatory effects consistent with secondary infection. ${ }^{27}$ Memory $B$ cells are primed to produce antibodies by the expression of IL-10, ${ }^{28}$ and with its proteolytic properties, IL-10 inhibited IFNy expression and deregulated the expressions of IL-6, IL-8, and TNF $\alpha$ in our study subjects and as previously described. ${ }^{29}$ Unlike previous reports that showed an association of IL-4 and disease severity, ${ }^{30}$ our study failed to reveal such findings probably because IL-4 was detected in only a few cases partly because of the small number of study subjects.

We demonstrated endothelial activation by the detection of IL-6, IL-8, and TNFa as previously described. ${ }^{3,10}$ This activation of the endothelial system not only contributes to bleeding, which is common in DF and DHF, ${ }^{11}$ but also involved in liver damage, leading to hepatitis complicating dengue. ${ }^{12}$ Our results were consistent with previous studies in respect to higher viremia in $\mathrm{DHF},{ }^{31}$ and we observed more bleeding and symptoms of systemic illness in DHF, reflecting the degree of severity when compared with DF. However, we did not find any association between viremia and bleeding as previously reported. ${ }^{32}$ Interleukin 8 is expressed by hepatocytes in dengue infection ${ }^{16}$ and was associated with disease severity. ${ }^{17}$ Similar to a previous study, ${ }^{33}$ our results regarding IL-8 levels could not distinguish DF from DHF. However, we demonstrated significantly elevated levels of IL-8 during the acute phase and at defervescence in patients with bleeding and in those with 
DF

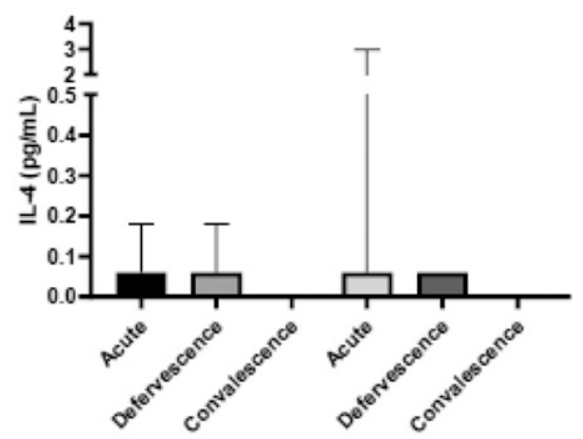

No Bleeding

Bleeding
DF

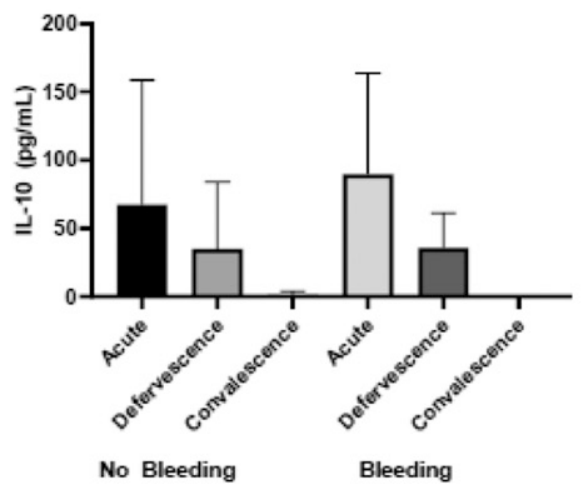

DF

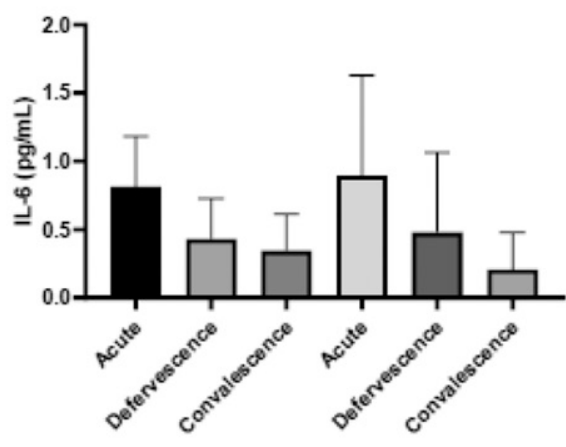

No Bleeding

Bleeding
DF

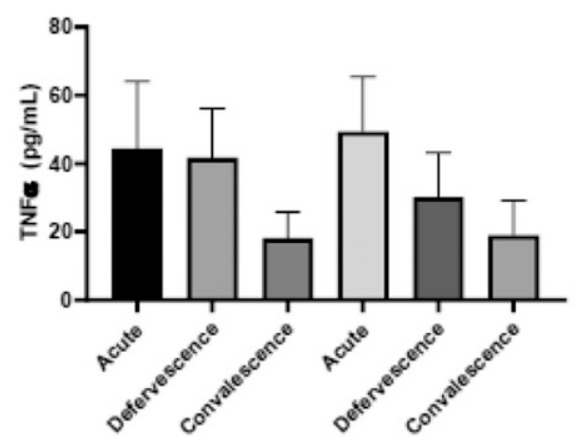

No Bleeding

Bleeding

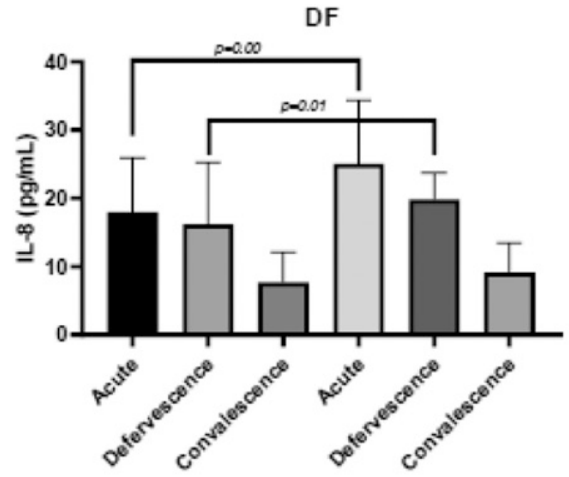

No Bleeding

FIGURE 6. Cytokine profile for bleeding in dengue fever during the three phases of infection.

hepatitis. We also showed an association of an increased IL-8 level with bleeding during both phases. Significantly, elevated levels of IL- 6 were observed during the acute phase in patients with bleeding, with a background of plasma leakage, whereas others reported elevated levels of IL-6 in DHF ${ }^{34}$ and bleeding. ${ }^{32}$ Other studies reported TNFa was not detectable or only detected in $30 \%$ of cases of dengue infection. ${ }^{35}$ In our study, we detected TNFa expression in all our subjects. We highlighted other factors that contribute to bleeding in dengue, such as thrombocytopenia, $^{36}$ which can worsen bleeding in dengue infection.

The liver impairment, which occurs in dengue infection, ${ }^{14}$ impacts bleeding with a worsening of coagulation. Our results demonstrated that IL-8 and TNFa expressions were significantly higher in patients with transaminases elevated 10-fold. Furthermore, we observed that IL-8 levels were significantly elevated with a 100-fold increase in transaminase levels during both phases.

The properties of IL- 8 contribute to platelet activation and endothelial permeability to cause thrombocytopenia, ${ }^{34}$ which worsens bleeding in dengue infection. We also observed that the platelet counts during both phases were significantly decreased in patients with bleeding. These elevated levels of IL-8 observed in our study might facilitate the events preceding bleeding during dengue infection. A similar observation of elevated levels of IL-8 with thrombocytopenia in dengue was described. ${ }^{34}$ During dengue infection, infected hepatocytes express IL-8, attracting neutrophils to the liver, causing liver injury and impaired coagulation. Our results show increased levels of neutrophils during the two phases of infection with hepatitis. This increase in neutrophils with hepatitis might be a result of the elevated levels of IL- 8 with hepatitis. Although a positive correlation of IFNy and transaminase was reported, ${ }^{34}$ we observed no similar pattern of correlations. Nevertheless, there were positive correlations between IL- 6 and IL- 8 and between IL- 6 and IFNy during the acute phase, and at defervescence IL-8 and TNFa were positively correlated, indicating these cytokines may act in synergy. In summary, the immunoregulatory responses observed may be implicated in the bleeding and hepatitis observed in dengue patients.

\section{CONCLUSION}

In this study, higher viremia in DHF with a T-helper 2 response determined by profiling the cytokine expression was demonstrated. The expression of IL-8 was significantly elevated during the acute phase in patients with bleeding in both DF and DHF, with a further increase in IL-8 levels in DHF at defervescence. IL-6 acts in synergy with IL-8 for bleeding with plasma leakage during the acute phase. Significantly, higher levels of TNF $\alpha$, IL-6, and IL-8 were observed in cases of severe hepatitis.

Limitations. There were some limitations in this study including the small number of total study subjects and those with severe disease. 
Dengue Infection

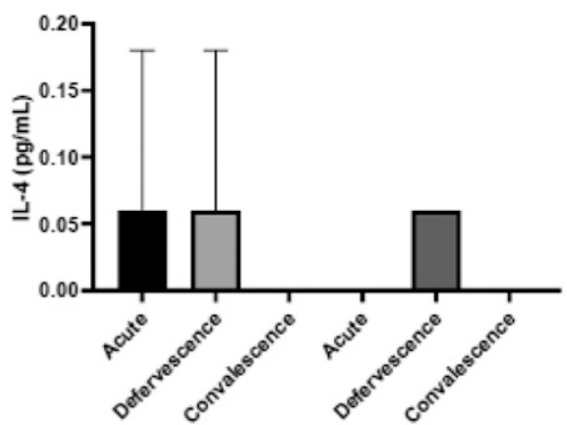

Non-severe Hepatitis Severe Hepatitis

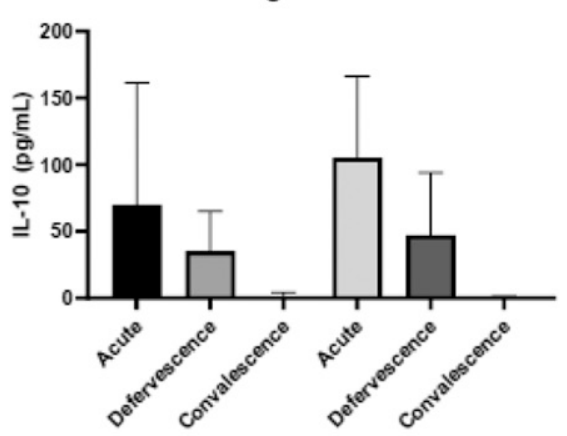

Non-severe Hepatitis Severe Hepatitis

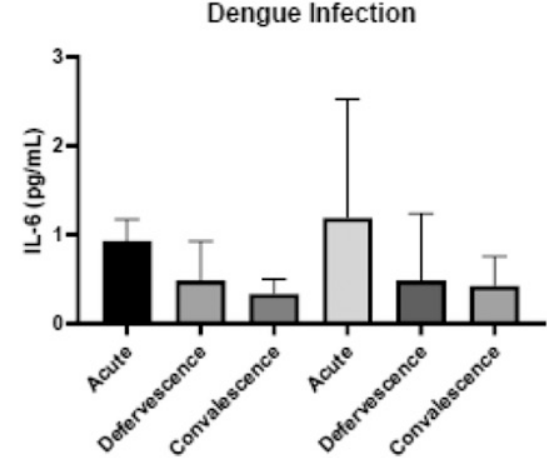

Non-severe Hepatitis Severe Hepatitis

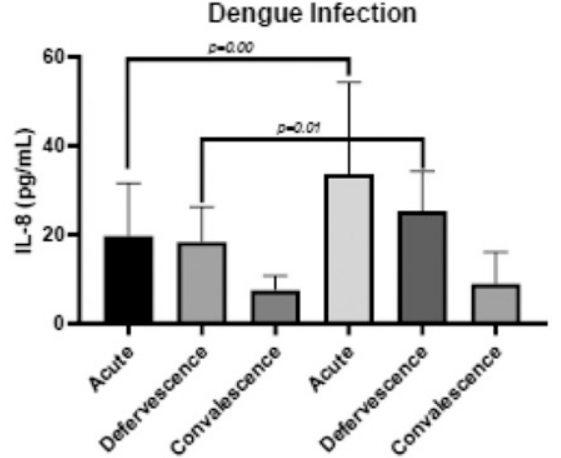

Non-severe Hepatitis Severe Hepatitis

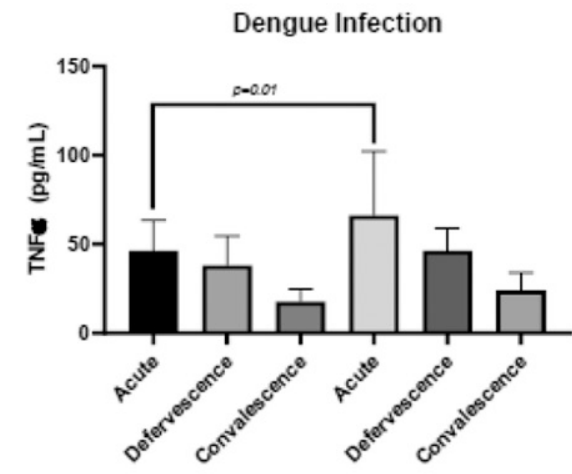

Non-severe Hepatitis Severe Hepatitis
Dengue Infection

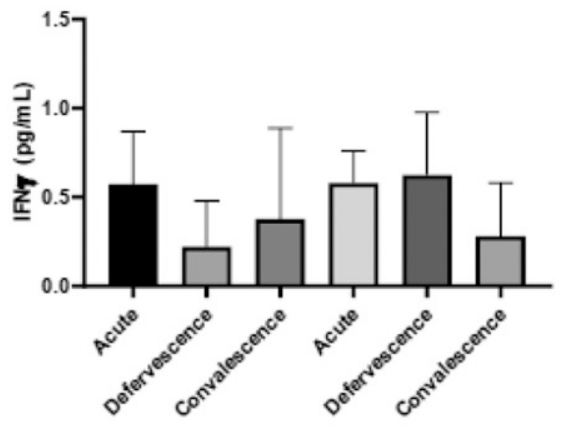

Non-severe Hepatitis Severe Hepatitis

FIGURE 7. Cytokine profile during the three phases of infection for severe hepatitis (AST/ALT $\geq 400 \mathrm{U} / \mathrm{L}$ ).

Received June 28, 2019. Accepted for publication January 19, 2020.

Published online March 2, 2020.

Acknowledgments: We thank the staff at Bangkok Hospital for Tropical Diseases for the support provided in this study and Jaranit Kaewkungwal and Pimphen Choroen for their assistance with the statistical analysis. We also acknowledge Edanz Group (www. edanzediting.com/ac) for editing a draft of this manuscript.

Financial support: This study was funded by 2015 Dean MOCID/ BIKEN scholarships awarded by Professor Yaowalak Sukthana for PhD research. The BIKEN Endowed Department of Dengue Vaccine Development was established by endowment from the Research Foundation for Microbial Diseases of Osaka University, Osaka, Japan, to the Research Institute for Microbial Diseases, Osaka University, Osaka, Japan. This work was partially supported by the Japan Agency for Medical Research and Development (AMED) JP18fm0108003.

Authors' addresses: Hisham Ahmed Imad, Weerapong Phumratanaprapin, Benjaluck Phonrat, Kesinee Chotivanich, Prakaykaew Charunwatthana, and Sant Muangnoicharoen, Department of Clinical Tropical Medicine, Faculty of Tropical Medicine, Mahidol University, Bangkok, Thailand, E-mails: hisham.a.imad@gmail.com, weerapong.phu@mahidol.ac.th, benjaluck.pho@mahidol.ac.th, nok@ tropmedres.ac, prakaykaew.cha@mahidol.ac.th, and sant.mua@ mahidol.ac.th. Srisin Khusmith, Department of Microbiology and Immunology, Faculty of Tropical Medicine, Mahidol University, Bangkok, Thailand, E-mail: srisin.khu@mahidol.ac.th. Terapong Tantawichien, Division of Infectious Diseases, Department of Medicine, Chulalongkorn University, Bangkok, Thailand, E-mail: terapong_ tantawichien@hotmail.co. Juthamas Phadungsombat, MahidolOsaka Center for Infectious Diseases, Faculty of Tropical Medicine, Mahidol University, Bangkok, Thailand, E-mail: juthamasps@ gmail.com. Emi Nakayama and Tatsuo Shioda, Mahidol-Osaka Center for Infectious Diseases, Faculty of Tropical Medicine, Mahidol University, Bangkok, Thailand, and Research Institute for Microbial Diseases, Osaka University, Osaka, Japan, E-mails: emien@biken. osaka-u.ac.jp and shioda@biken.osaka-u.ac.jp. Eiji Konishi, BIKEN Endowed Department of Dengue Vaccine Development, Faculty of Tropical Medicine, Mahidol University, Bangkok, Thailand, E-mail: ekon@biken.osaka-u.ac.jp.

This is an open-access article distributed under the terms of the Creative Commons Attribution (CC-BY) License, which permits unrestricted use, distribution, and reproduction in any medium, provided the original author and source are credited.

\section{REFERENCES}

1. Gubler DJ, 2011. Dengue, urbanization and globalization: the unholy trinity of the 21(st) century. Trop Med Health 39 (Suppl 4): 3-11.

2. Lauer SA et al., 2018. Prospective forecasts of annual dengue hemorrhagic fever incidence in Thailand, 2010-2014. Proc Natl Acad Sci USA 115: E2175-E2182.

3. Huang YH, Lei HY, Liu HS, Lin YS, Liu CC, Yeh TM, 2000. Dengue virus infects human endothelial cells and induces IL-6 and IL-8 production. Am J Trop Med Hyg 63: 71-75.

4. Suksanpaisan L, Cabrera-Hernandez A, Smith DR, 2007. Infection of human primary hepatocytes with dengue virus serotype 2 . J Med Virol 79: 300-307.

5. Bosch I, Xhaja K, Estevez L, Raines G, Melichar H, Warke RV, Fournier MV, Ennis FA, Rothman AL, 2002. Increased production of interleukin-8 in primary human monocytes and in human epithelial and endothelial cell lines after dengue virus challenge. J Virol 76: 5588-5597.

6. Vervaeke P, Vermeire K, Liekens S, 2015. Endothelial dysfunction in dengue virus pathology. Rev Med virol 25: 50-67.

7. Chunhakan S, Butthep P, Yoksan S, Tangnararatchakit K, Chuansumrit A, 2015. Vascular leakage in dengue hemorrhagic fever is associated with dengue infected monocytes, monocyte activation/exhaustion, and cytokines production. Int $J$ Vasc Med 2015: 917143. 
8. Chaturvedi UC, Agarwal R, Elbishbishi EA, Mustafa AS, 2000. Cytokine cascade in dengue hemorrhagic fever: implications for pathogenesis. FEMS Immunol Med Microbiol 28: 183-188.

9. Halstead SB, Mahalingam S, Marovich MA, Ubol S, Mosser DM, 2010. Intrinsic antibody-dependent enhancement of microbial infection in macrophages: disease regulation by immune complexes. Lancet Infect Dis 10: 712-722.

10. Inyoo S, Suttitheptumrong A, Pattanakitsakul SN, 2017. Synergistic effect of TNF-alpha and dengue virus infection on adhesion molecule reorganization in human endothelial cells. Jpn $J$ Infect Dis 70: 186-191.

11. Thanachartwet V, Oer-Areemitr N, Chamnanchanunt S, Sahassananda D, Jittmittraphap A, Suwannakudt P, Desakorn V, Wattanathum A, 2015. Identification of clinical factors associated with severe dengue among Thai adults: a prospective study. BMC Infect Dis 15: 420.

12. Samanta J, Sharma V, 2015. Dengue and its effects on liver. World J Clin Cases 3: 125-131.

13. Rajapakse S, de Silva NL, Weeratunga P, Rodrigo C, Fernando SD, 2017. Prophylactic and therapeutic interventions for bleeding in dengue: a systematic review. Trans $R$ Soc Trop Med Hyg 111: 433-439.

14. Treeprasertsuk S, Kittitrakul C, 2015. Liver complications in adult dengue and current management. Southeast Asian J Trop Med Public Health 46 (Suppl 1): 99-107.

15. de Azeredo EL, Monteiro RQ, de-Oliveira Pinto LM, 2015. Thrombocytopenia in dengue: interrelationship between virus and the imbalance between coagulation and fibrinolysis and inflammatory mediators. Mediators Inflamm 2015: 313842.

16. Medin CL, Fitzgerald KA, Rothman AL, 2005. Dengue virus nonstructural protein NS5 induces interleukin-8 transcription and secretion. J Virol 79: 11053-11061.

17. Raghupathy $\mathrm{R}$ et al., 1998;Elevated levels of IL-8 in dengue hemorrhagic fever. J Med Virol 56: 280-285.

18. Nagila A, Netsawang J, Suttitheptumrong A, Morchang A, Khunchai S, Srisawat C, Puttikhunt C, Noisakran S, Yenchitsomanus PT, Limjindaporn T, 2013. Inhibition of p38MAPK and CD137 signaling reduce dengue virus-induced TNF-alpha secretion and apoptosis. Virol $J$ 10: 105.

19. Sehrawat $P$, Biswas A, Kumar P, Singla P, Wig N, Dar L, Sood R, 2018. Role of cytokines as molecular marker of dengue severity. Mediterr J Hematol Infect Dis 10: e2018023.

20. Shu PY, Chang SF, Kuo YC, Yueh YY, Chien LJ, Sue CL, Lin TH, Huang JH, 2003. Development of group- and serotype-specific one-step SYBR green I-based real-time reverse transcriptionPCR assay for dengue virus. J Clin Microbiol 41: 2408-2416.

21. Wang WK, Lee CN, Kao CL, Lin YL, King CC, 2000. Quantitative competitive reverse transcription-PCR for quantification of dengue virus RNA. J Clin Microbiol 38: 3306-3310.

22. Lin $\mathrm{CY}$, Huang $\mathrm{CH}$, Chen $\mathrm{YH}, 2013$. Classification of dengue: the clinical use of World Health Organization 2009 guideline. J Formos Med Assoc 112: 61-63.
23. Munoz-Jordan JL, Sanchez-Burgos GG, Laurent-Rolle M, Garcia-Sastre A, 2003. Inhibition of interferon signaling by dengue virus. Proc Natl Acad Sci USA 100: 14333-14338.

24. Silverman RH, 2007. Viral encounters with 2',5'-oligoadenylate synthetase and RNase $\mathrm{L}$ during the interferon antiviral response. J Virol 81: 12720-12729.

25. Chakravarti A, Kumaria R, 2006;Circulating levels of tumour necrosis factor-alpha \& interferon-gamma in patients with dengue \& dengue haemorrhagic fever during an outbreak. Indian J Med Res 123: 25-30.

26. Morrison J, Aguirre S, Fernandez-Sesma A, 2012. Innate immunity evasion by dengue virus. Viruses 4: 397-413.

27. Maneekan P, Leaungwutiwong P, Misse D, Luplertlop N, 2013. T helper (Th) 1 and Th2 cytokine expression profile in dengue and malaria infection using magnetic bead-based bio-plex assay. Southeast Asian J Trop Med Public Health 44: 31-36.

28. Malavige GN, Gomes L, Alles L, Chang T, Salimi M, Fernando S, Nanayakkara KD, Jayaratne S, Ogg GS, Serum IL-10 as a marker of severe dengue infection. BMC Infect Dis 2013;13: 341.

29. Couper KN, Blount DG, Riley EM, 2008. IL-10: the master regulator of immunity to infection. J Immunol 180: 5771-5777.

30. Abhishek KS, Chakravarti A, Baveja CP, Kumar N, Siddiqui O, Kumar S, 2017. Association of interleukin-2, -4 and -10 with dengue severity. Indian J Pathol Microbiol 60: 66-69.

31. Vaughn DW et al., 2000. Dengue viremia titer, antibody response pattern, and virus serotype correlate with disease severity. $J$ Infect Dis 181: 2-9.

32. Iani FC, Caldas S, Duarte MM, Cury AL, Cecílio AB, Costa PA, Antonelli LR, Gollob KJ, 2016. Dengue patients with early hemorrhagic manifestations lose coordinate expression of the anti-inflammatory cytokine IL-10 with the inflammatory cytokines IL-6 and IL-8. Am J Trop Med Hyg 95: 193-200.

33. Cruz Hernandez SI, Puerta-Guardo HN, Flores Aguilar $\mathrm{H}$, González Mateos S, López Martinez I, Ortiz-Navarrete V, Ludert JE, Angel RM, 2016. Primary dengue virus infections induce differential cytokine production in Mexican patients. Mem Inst Oswaldo Cruz 111: 161-167.

34. Priyadarshini D, Gadia RR, Tripathy A, Gurukumar KR, Bhagat A, Patwardhan S, Mokashi N, Vaidya D, Shah PS, Cecilia D, 2010, Clinical findings and pro-inflammatory cytokines in dengue patients in western India: a facility-based study. PLoS One 5: e8709.

35. Pinto LM, Oliveira SA, Braga EL, Nogueira RM, Kubelka CF, 1999. Increased pro-inflammatory cytokines (TNF-alpha and IL-6) and anti-inflammatory compounds (sTNFRp55 and sTNFRp75) in Brazilian patients during exanthematic dengue fever. Mem Inst Oswaldo Cruz 94: 387-394.

36. Rothman AL, Ennis FA, 1999. Immunopathogenesis of dengue hemorrhagic fever. Virol 257: 1-6. 